Article

\title{
Sensitivity of Discrete Symmetry Tests in the Positronium System with the J-PET Detector
}

\author{
Aleksander Gajos (D) \\ Faculty of Physics, Astronomy and Applied Computer Science, Jagiellonian University, S. Łojasiewicza 11, \\ 30-348 Kraków, Poland; aleksander.gajos@uj.edu.pl
}

Received: 1 June 2020; Accepted: 2 July 2020; Published: 1 August 2020

\begin{abstract}
Study of certain angular correlations in the three-photon annihilations of the triplet state of positronium, the electron-positron bound state, may be used as a probe of potential CP and CPT-violating effects in the leptonic sector. We present the perspectives of CP and CPT tests using this process recorded with a novel detection system for photons in the positron annihilation energy range, the Jagiellonian Positron Emission Tomography (J-PET). We demonstrate the capability of this system to register three-photon annihilations with an unprecedented range of kinematical configurations and to measure the CPT-odd correlation between positronium spin and annihilation plane orientation with a precision improved by at least an order of magnitude with respect to present results. We also discuss the means to control and reduce detector asymmetries in order to allow J-PET to set the first measurement of the correlation between positronium spin and momentum of the most energetic annihilation photon which has never been studied to date.
\end{abstract}

Keywords: discrete symmetry; CPT; positronium

\section{Introduction}

The notion of searching of for violation of fundamental discrete symmetries in purely leptonic systems and in electromagnetic interactions, although not new, has been outside of the mainstream of symmetry tests in physics for a few decades after the first violation had been discovered.

While the pioneering discoveries naturally led the attention towards weak interactions [1,2], other violation mechanisms should not be ruled out precipitately.

A viable purely leptonic system for tests of discrete symmetries is constituted by positronium exotic atoms. As a bound state of electron and positron, positronium is the lightest matter-antimatter system and at the same time an eigenstate of the $\mathrm{C}$ and $\mathrm{P}$ operations, making it an ideal candidate for searching of symmetry violating effects [3]. This potential has been recognized already in 1967 by Mills and Berko, who performed a search for decays of annihilations of the positronium C-even singlet state into a C-odd three-photon final state, concluded with a null result [4].

The field of discrete symmetry studies in the lepton sector has seen little activity until Bernreuther et al. pointed out that violations of the CP and CPT symmetries could be manifested by certain non-vanishing angular correlations in the decays of positronium atoms if the corresponding operators constructed with observables available in the decay are odd under a given symmetry transformation [5]. Several implementations of tests based on such angular correlations followed, with the best measurements to date yielding results consistent with conservation of both $\mathrm{CP}$ and $\mathrm{CPT}$ with precision at the level of $10^{-3}[6,7]$. Notably, authors of the most recent CPT test performed using the Gammasphere array of germanium detectors have also extended the searches for $C$ violation by searching for higher-order C-prohibited annihilations of positronium [8]. Other prohibited positronium decays were studied to test lowest-order QED calculations [9,10]. 
Since the discovery of neutrino oscillations, searches for leptonic $\mathrm{CP}$ violation were strongly concentrated on neutrino physics [11]. Although other tests were attempted e.g., by searching for the electric dipole moment of $\tau$ [12], it is indeed the long-baseline neutrino oscillation experiments which first show hints of observation of CP violation at $3 \sigma$ level [13-15].

The interest in positronium as a potential probe of CPT violation has been recently revived by the postulation of possible effects of Lorentz invariance observable with positronium in the framework of the Standard Model Extension (SME). SME is a general-realistic effective field theory of Lorentz violation, which extends systems' Lagrangians to include all effectively possible Lorentz-violating terms. The inherent relation of Lorentz and CPT invariance allows for defining searches of violations of the latter in terms of SME parameters' measurement. A number of possible experiments based on hyperfine spectroscopy of positronium have been postulated using both minimal SME [16] and non-minimal SME scenarios [17].

The Jagiellonian Positron Emission Tomography (J-PET) collaboration strives to explore an experimental program complementary to the SME-motivated spectroscopic studies. Exploiting the potential of a novel powerful detector of photons in the positron annihilation energy range, we aim at extending the measurements of angular correlations in the decays of the positronium triplet state, which are sensitive to effects violation of fundamental symmetries [3]. In this work, we present the scope of experimental CP and CPT tests available with the J-PET detector based on large-acceptance exclusive detection of ortho-positronium annihilations and an unconventional scheme of positronium spin orientation estimation on a single-event basis.

\section{The J-PET Detector}

J-PET was conceived as the first Positron Emission Tomography (PET) scanner based on plastic scintillators [18-21]. While actively exploited in medical imaging research towards constructing a cost-effective whole-body PET scanner [20,22,23] and devising new imaging modalities such as spatially-resolved determination of properties positronium atoms produced during a PET scan [24,24-26], J-PET also constitutes a robust detector of photons in the sub-MeV range, well suited for studies of phenomena such as positronium annihilation and entanglement of photons in the field of fundamental research [27-29].

The core of the detector is constituted by 192 photon detection modules sparsely arranged in three concentric layers along the longitudinal axis of the detector as presented in the left panel of Figure 1. Each module consists of an EJ-230 plastic scintillator strip of $50 \mathrm{~cm}$ length and $7 \times 19 \mathrm{~mm}^{2}$ cross-section, whose both ends are optically coupled to Hamamatsu R9800 photomultiplier tubes.

Interactions of photons in the plastic scintillators are recorded through their Compton scattering resulting in an energy deposition depending on the scattering angle and emission of scintillation light recorded by the two photomultipliers. Time of interaction and its position along the strip are determined using time difference between light recording at the two ends of the strip. Lack of registration of the full energy peak in J-PET detection modules is compensated by an excellent interaction time resolution at the level of 100 ps [18] resulting from fast front-end electronics [30] and short decay times of plastic scintillators. Additionally, the latter allows for pileup-free measurements with high positron source activities of $10 \mathrm{MBq}$ and more.

Electric signals from the photomultipliers are sampled in the voltage domain at four configurable voltage thresholds by scalable front-end electronics developed for J-PET coupled with a data acquisition (DAQ) system based on Field Programmable Gate Array (FPGA) chips and the TRB3 platform [30-32]. The DAQ of J-PET is reconfigurable and opens the possibilities of real-time data reconstruction directly on the FPGA systems [33]. 


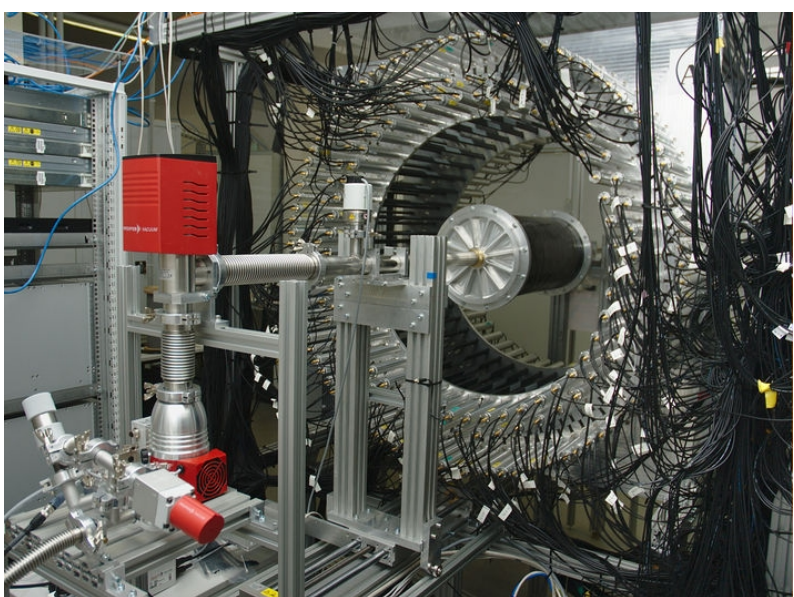

(a)

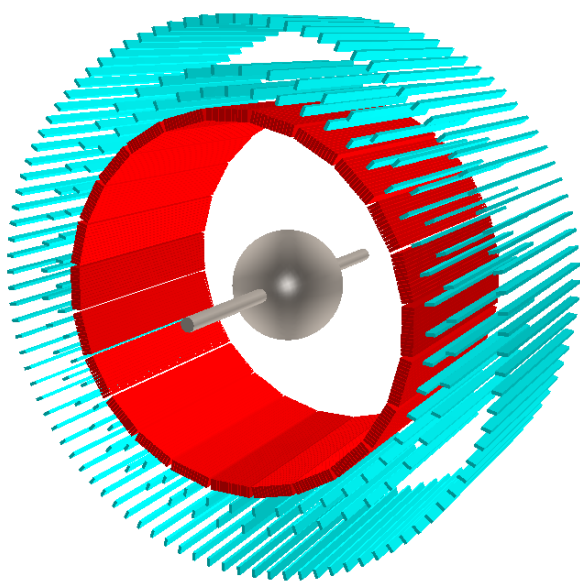

(b)

Figure 1. (a): View of the Jagiellonian Positron Emission Tomography (J-PET) detector with a cylindrical vacuum chamber for positronium production and annihilation mounted in its center. (b): Schematic view of two future extensions of the experimental setup: (i) the current three layers of sparsely-arranged scintillator strips (blue) will be complemented by a layer of 24 modules containing 13 densely-packed scintillator strips each (red); (ii) the cylindrical annihilation chamber will be replaced by a spherical one (gray).

Energy deposited by a $\gamma$ quantum interacting in a scintillator strip is measured through total charge of the electric signals from the attached photomultipliers, estimated by the signals' time-over-threshold sampled at the four predefined voltages. Thanks to the ability of J-PET to record Compton scattering angles in multiple scattering events, deposited energy corresponding unanimously to a given angle is related to the recorded time over threshold allowing for calibration of deposited energy measurement [34,35].

The scope of applications of the detector ranging from medical imaging development to fundamental studies of positronium annihilations (including modes not observed to date such as ortho-positronium (o-Ps) annihilation into $4 \gamma$ [3]) requires the DAQ system to impose a minimal bias on the spectrum of recorded events [36]. This is achieved with recording of data in a trigger-less mode [32,37] followed by filtering and reconstruction with a dedicated analysis software framework [38-40]. While an unusual choice due to large resulting data volume, in the case of searches for small effects such as rare decays and symmetry violations which can be easily mimicked by a non-uniform response of the detector and DAQ elements such as trigger bias imposed on the data, only complete foregoing of the trigger allows for full control over systematic effects in the experiments.

Currently, J-PET is being extended with an additional layer of detection modules organized in a dense layout placed within the current setup as displayed schematically in the right panel of Figure 1. These modules are intended to enhance the angular acceptance of the detector as well as to provide an improved time resolution thanks to an entirely digital readout using matrices of silicon photomultipliers [41]. The impact of this extension of the detection setup on the discrete symmetry tests' sensitivity is discussed in Section 4.

\section{Methods of Searching for Discrete Symmetry Violations with Ortho-Positronium in J-PET}

The ability to record photons in an energy range corresponding to electron-positron annihilations as well as below it makes J-PET a suitable device for studying decays of the lightest purely leptonic bound system, the positronium exotic atom. Positronium, the bound state of electron and positron, may be formed as a singlet or triplet ground state, referred to as para-positronium (p-Ps) and ortho-positronium (o-Ps), respectively. Being an antisymmetric eigenstate of charge conjugation, the latter may only annihilate into an odd number of photons due to the conservation of the $C$ symmetry, 
tested to the level of $10^{-6}$ for positronium [8,9]. In practice, ortho-positronium predominantly annihilates into a three-photon final state with the next allowed final state $(5 \gamma)$ suppressed by a factor of $\alpha^{2}$.

While the positronium physics appears to be well described by electromagnetic interactions where $\mathrm{CP}$ violation is not expected, any observation of $\mathrm{CP}$ noninvariance in this system would be an indication of new physics. Motivation for such searches is further encouraged by the recent neutrino oscillation measurements hinting at leptonic $\mathrm{CP}$ violation at $3 \sigma$ level [13-15], for which no confirmation was provided by charged lepton systems to date. As pointed out by Bernreuther and Nachtmann [5], the three-photon annihilations of the triplet state of positronium may provide insight into $\mathrm{CP}$ and even CPT-violating effects through certain angular correlations between o-Ps spin and momenta of annihilation photons.

Table 1 presents three angular correlations measurable in the ortho-positronium three-photon annihilations. The correlations are represented as operators whose properties under the $\mathrm{C}, \mathrm{P}$ and $\mathrm{T}$ transformations and their combinations follow from the respective behavior of positronium spin $(\vec{S})$ and momentum vectors of the final state photons $\left(\vec{k}_{i}\right.$ for $i=1,2,3$ where the photons are labeled according to descending energy, i.e., $\left|\vec{k}_{1}\right|>\left|\vec{k}_{2}\right|>\left|\vec{k}_{3}\right|$ ) under these operations. In case of operators which are antisymmetric under a given transformation (marked with " - " in the table), expectation value of the operator must vanish if the respective transformation constitutes a good symmetry. Consequently, observation of a non-zero expectation value of such an operator would be an indication of violation of a given discrete symmetry $[5,36]$. The notion of testing discrete symmetries in the annihilations of ortho-positronium is therefore based on experimental determination of the expectation values of the angular correlation operators listed in Table 1. Notably, only one experiment conducted to date attempted to probe a continuous distribution of such expectation values [7] whereas all previous measurements were constrained to determination of an up-down asymmetry of the operators, a special case with significantly limited sensitivity $[6,42,43]$.

Table 1. Angular correlation operators constructed with observables of ortho-positronium annihilations into three photons: positronium spin $\vec{S}$ and momenta of the annihilation photons ordered by their magnitude: $\left|\vec{k}_{1}\right|>\left|\vec{k}_{2}\right|>\left|\vec{k}_{3}\right|$. Each of these operators is either even (+) or odd (-) with respect to the basic symmetry transformations and their combinations as marked in the table. While measurement of operators 1 and 2 only requires non-zero vector polarization of ortho-positronium, operator 3 is sensitive to $\mathrm{CP}$ violation in the presence of a specific tensor polarization $[5,44]$ produced e.g., by an external magnetic field [42].

\begin{tabular}{ccccccc}
\hline No. & Operator & C & P & T & CP & CPT \\
\hline 1 & $\vec{S} \cdot \overrightarrow{k_{1}}$ & + & - & + & - & - \\
2 & $\vec{S} \cdot\left(\overrightarrow{k_{1}} \times \overrightarrow{k_{2}}\right)$ & + & + & - & + & - \\
3 & $\left(\vec{S} \cdot \overrightarrow{k_{1}}\right)\left(\vec{S} \cdot\left(\overrightarrow{k_{1}} \times \overrightarrow{k_{2}}\right)\right)$ & + & - & - & - & + \\
\hline
\end{tabular}

Operators 1 and 2 presented in Table 1 are sensitive to CPT-violating effects if the annihilating positronium spin is known, e.g., due to vector polarization originating from positron polarization inherent to $\beta^{+}$decay. While the third CP-odd operator appears as a simple product of the former two, its definition involves positronium spin twice in an anti-symmetric combination $[5,44]$ and is only measurable in presence of more specific tensor polarization, calling for experimental approaches distinct from that available for operators 1 and 2, such as positronium polarization using an external magnetic field. In this work, we focus on the capabilities of the J-PET experiment in conducting precise measurements of the first two of the presented angular correlations, using a spin estimation technique without external magnetic field and probing full geometrically-allowed domains of the correlation operators for the first time. 


\subsection{Estimation of Positronium Spin}

An essential component of the angular correlations in positronium decays considered in this work is the knowledge of the positronium spin quantization axis. Former measurements either used a polarized positronium beam [43], external magnetic field [6,42] or relied on the intrinsic linear polarization of positrons emitted in $\beta^{+}$decay [7]. The two former approaches exclusively allow for producing a degree of tensor polarization in the positronium sample, inevitable for conducting a test of the CP symmetry with operator no. 3 from Table 1 . However, setups required to convey the beam to the annihilation recording device and magnets providing sufficient $\vec{B}$ field effectively prevent recording of the annihilation photons with a large angular acceptance.

Therefore, J-PET builds on the o-Ps polarization control scheme proposed in the best measurement of the $\vec{S} \cdot\left(\overrightarrow{k_{1}} \times \overrightarrow{k_{2}}\right)$ operator to date [7], in which longitudinally-polarized positrons from a point-like $\beta^{+}$source of ${ }^{68} \mathrm{Ge}$ or ${ }^{22} \mathrm{Na}$ are allowed to form positronia only in a limited volume which defines a range of allowed $e^{+}$spin quantization axes. As the positron polarization statistically translates to the formed ortho-positronium in $2 / 3$ of cases, this allows for obtaining an estimate of the o-Ps spin direction with a finite uncertainty determined by the $\beta^{+}$emission average energy and the applied geometry of positronium formation medium. In the original implementation of this idea, the latter uncertainty accounted for a reduction of statistical polarization by 0.686 , in addition to the average polarization of $P \approx 0.4$ inherent to the method of producing ortho-positronium [7]. On the other hand, it evaded the need for a acceptance-limiting hardware setup which allowed for the first measurement of a true distribution of an angular correlation in o-Ps annihilation, although limited in resolution by the coarse detector granularity.

In the measurements with J-PET proposed in this work, we extend the idea of estimating ortho-positronium spin without externally-induced polarization. While it limits the accessible symmetry violating operators to positions 1 and 2 from Table 1 as measurement of the correlation $\left(\vec{S} \cdot \overrightarrow{k_{1}}\right)\left(\vec{S} \cdot\left(\overrightarrow{k_{1}} \times \overrightarrow{k_{2}}\right)\right)$ is only possible in the case of a tensor-polarized positronium sample [45], this allows J-PET to observe an unprecedented spectrum of angular configurations of o-Ps decays and thus the full spectra of correlation operators 1 and 2.

To this end, we use positrons emitted from a point-like $\beta^{+}$source which are characterized by linear polarization along their direction of emission to a degree of $P_{e^{+}}=v / c$ where $v$ is the positron velocity and $c$ is the speed of light. The positrons are allowed to thermalize in a layer of porous medium enhancing positronium formation, which is spatially separated from the $\beta^{+}$source by the volume of vacuum chamber ensuring free propagation of the positrons.

In contrast to the previous measurement [7], we do not assume the positronium production region to be point-like but use the information on the locations and times of the three photons' interactions in the detector to reconstruct the o-Ps $\rightarrow 3 \gamma$ annihilation point with a trilateration-based approach [46]. In consequence, we can estimate the direction of $e^{+}$spin separately for each event, thus reducing the related decrease in statistical o-Ps polarization from 0.686 to about 0.98 determined by the spatial resolution of the o-Ps $\rightarrow 3 \gamma$ reconstruction.

In the currently performed measurements, J-PET implements the aforementioned spin estimation scheme with a cylindrical positronium production chamber mounted axially in the center and extending for the whole length of the detector. A $10 \mathrm{MBq} \beta^{+}$source of ${ }^{22} \mathrm{Na}$ is installed in the center of the chamber, while its walls are coated with $3 \mathrm{~mm}$ of R60G porous silica, allowing practically all positrons reaching the chamber walls to thermalize and interact within this layer [47]. The chamber walls are made of $3 \mathrm{~mm}$ polycarbonate so as to minimize absorption and scattering of annihilation photons. The chamber mounted inside the J-PET detector is presented in the left panel of Figure 1. The right panel of the figure illustrates a future enhancement of the chamber geometry, i.e., replacement of the cylinder with a spherical vacuum chamber (with $R=10 \mathrm{~cm}$ ), which allows for a more efficient utilization of positrons from the $\beta^{+}$source for positronium formation, increases o-Ps $\rightarrow 3 \gamma$ registration efficiency for extreme values of certain correlations and reduces spurious asymmetries as demonstrated in the next sections. 


\subsection{The Correlation between o-Ps Spin and Annihilation Plane}

The 2nd operator from Table 1 is sensitive to potential violations of CPT invariance and has been previously studied in two experiments with the most precise result of the CPT-violation parameter $C_{C P T}$ of $(2.6 \pm 3.1) \times 10^{-3}$ [7,43]. In fact, a similarly-defined triple correlation has been studied in search for $T$ violation in decays using $Z^{0}$ spin and momenta of the most energetic produced jets [48].

As can be seen from Table 1 , the $\vec{S} \cdot \overrightarrow{k_{1}}$ correlation is also odd under the CPT transformation. The choice of the ostensibly more complicated operator in the previous measurements was motivated by the fact that $\vec{S} \cdot\left(\overrightarrow{k_{1}} \times \overrightarrow{k_{2}}\right)$ contains a simple correlation between the o-Ps spin and positronium annihilation plane spanned by the momentum vectors of the emitted photons. The definition using the two most energetic photons' momenta is merely an experimentally-useful convention and does not introduce a significant correlation between detection efficiency and photon energy as is the case for the $\vec{S} \cdot \overrightarrow{k_{1}}$ operator as argued later on in this work. In order to avoid any dependence on selected photon energies, it is convenient to normalize this operator to the magnitude of the cross product, leading to the following definition:

$$
\mathcal{O}_{C P T}=\hat{S} \cdot\left(\vec{k}_{1} \times \vec{k}_{2}\right) /\left|\vec{k}_{1} \times \vec{k}_{2}\right|,
$$

which expresses the pure angular correlation between o-Ps spin and its decay plane.

The best measurement to date was simultaneously the first measurement going beyond the up-down asymmetry of the operator and determining its continuous distribution. However, due to the geometry of the Gammasphere detector used therein and the positronium production setup, the measurement was only sensitive to the values of this operator in the range of about $(-0.4,0.4)$ out of the allowed region of $[-1,1]$.

Due to its high granularity of the detection modules in the transverse plane and continuous interaction position measurement in the longitudinal coordinate, in conjunction with the spin estimation scheme which does not impose a distinguished positronium spin quantization axis with respect to the detector, the J-PET setup is able to record a substantially broader range of kinematic configurations of o-Ps $\rightarrow 3 \gamma$ events.

To demonstrate the sensitivity of J-PET to the distribution of the $\mathcal{O}_{C P T}$ operator, a toy Monte Carlo simulation of ortho-positronium annihilations in the experimental setup described above was prepared, featuring allowed angular and energy distributions of photons from o-Ps $\rightarrow 3 \gamma$ annihilations expected from QED [49], the geometry of the positronium production setup as well as geometric arrangement of the detection modules. Compton interactions of the annihilation photons were simulated according to the Klein-Nishina formula and a photon registration threshold was set on the simulated deposition of energy by the scattered photon in a scintillator strip.

The distribution of the $\mathcal{O}_{C P T}$ operator, i.e., cosine of the angle between the normal to the annihilation plane and positronium spin direction was simulated either to be uniform as expected in the absence of CPT-violating effects [5] or with an assumed level of asymmetry quantified by a $C_{C P T}$ coefficient. Following the approach used in Ref. [7], the simplest asymmetric form of the distribution as a function of $\cos \theta$ was used where the total probability distribution contains a term linear in $\cos \theta$ whose contribution given by $C_{C P T} \in[0 ; 1]$.

A simulation of $10^{13}$ positrons emitted from a $\beta^{+}$source without CPT-violating effects in ortho-positronium annihilations results in a distribution of the $\mathcal{O}_{C P T}$ operator presented in the hatched blue histogram presented in Figure 2. Red histogram in the same figure corresponds to the distribution obtained if maximal violation of the symmetry $\left(C_{C P T}=1\right)$ is assumed. While the existing measurements exclude values of $C_{C P T}$ beyond the $10^{-3}$ level, the exaggeration in Figure 2 was used to visualize the effects detectable through determination of the distribution of this angular correlation. It is visible that while detection efficiency peaks for values corresponding to the decay plane normal being close to perpendicular to the spin quantization axis, it does not drop to zero close to the extreme values of the correlation, in contrast to the previous measurement [7]. 


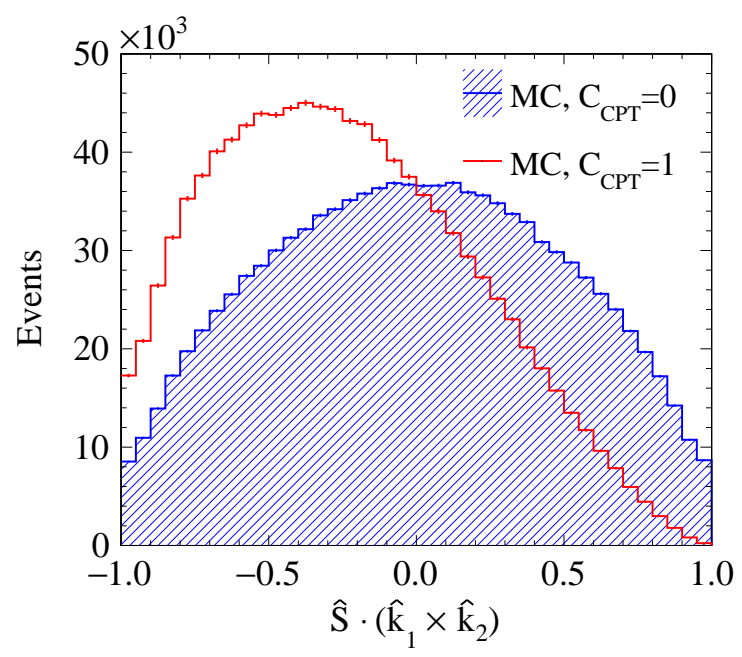

Figure 2. Distributions of the $\mathcal{O}_{C P T}$ operator resulting from toy Monte Carlo simulations of $10^{13}$ positron interactions in J-PET with the cylindrical positronium production chamber in case of no $\mathrm{CPT}$ violation assumed in the simulation (hatched blue histogram) and extreme violation (hollow red histogram).

The factors determining the detection efficiency of o-Ps $\rightarrow 3 \gamma$ events in J-PET comprise (i) probability of interaction of an annihilation photon in a plastic scintillator strip which on average amounts to about $20 \%$, (ii) geometrical acceptance resulting from the sparse arrangement of detection modules and their length; modules cover about 0.21 of the solid angle around the center of the detector, (iii) the energy deposition threshold above which a Compton-scattered photon is registered by a detection module. Furthermore, the total efficiency of observing ortho-positronium annihilations as a function of the $\beta^{+}$source activity also involves (iv) the fraction of positrons forming o-Ps in the region of the annihilation chamber where the three emitted photons can be recorded simultaneously.

Figure 3a presents the total o-Ps $\rightarrow 3 \gamma$ registration efficiency as a function of the angular correlation defined in Equation (1) obtained in toy MC simulation of $10^{13}$ positrons from a ${ }^{22}$ Na source in the setup described in Section 3.1. The efficiency is presented for two geometries of the annihilation chamber: cylindrical (presently used) and spherical (in preparation). In each case, three values of the energy deposition threshold for a single annihilation photon were considered: $40 \mathrm{keV}, 100 \mathrm{keV}$ and $140 \mathrm{keV}$. Results of the simulation show that lowering this photon registration threshold is vital for the total efficiency and each increase of the threshold by about $50 \mathrm{keV}$ results in a reduction of the $3 \gamma$ registration efficiency by an order of magnitude.

Presently, the detection threshold of the J-PET photomultiplier tubes and signal sampling electronics achievable without entering the noise level is estimated to be about $80 \mathrm{keV}$.

The MC-based evaluation of detection efficiency also demonstrates the enhancement expected with the spherically-shaped positronium production vacuum chamber instead of the currently used cylindrical one. The drop of efficiency close to the extreme values of $\mathcal{O}_{C P T}$ visible in Figure 2 will be substantially reduced with the new source geometry, resulting in an efficiency more flat across the whole operator spectrum.

A subtle asymmetry in the distribution of a given angular correlation $\mathrm{X}$ may be detected by evaluation of the following figure, accounting for asymmetry between event counts $N$ in subsequent intervals of for positive and negative values of a given angular correlation operator $\mathcal{O}_{X}$ :

$$
A\left(\left|\mathcal{O}_{X}\right|\right)=\frac{N\left(\left|\mathcal{O}_{X}\right|\right)-N\left(-\left|\mathcal{O}_{X}\right|\right)}{N\left(\left|\mathcal{O}_{X}\right|\right)+N\left(-\left|\mathcal{O}_{X}\right|\right)}
$$

Subsequently, a comparison of the $A\left(\left|\mathcal{O}_{C P T}\right|\right)$ distribution with one obtained in case of the MC-simulated distribution assuming maximal violation $\left(C_{C P T}=1\right)$ would allow for extraction of 
the CPT symmetry violation coefficient in a similar manner as done e.g., in Ref. [7]. For such a procedure, good understanding of the detector efficiency as a function of the value of the measured operator is crucial in order to avoid artificial asymmetries arising from efficiency nonuniformities due to the setup geometry. In J-PET, thanks to the large granularity of detection modules and continuous measurement of interaction positions along them, the expected shape of such efficiency is smooth as demonstrated in the left panel of Figure 3. While this is already an improvement with respect to the previous measurement of the $\mathcal{O}_{C P T}$ operator where coarse granularity of the detectors constituting the Gammasphere array caused strong periodic fluctuations of efficiency [7], the impact of detector geometry on the measured asymmetry requires a careful treatment nonetheless.

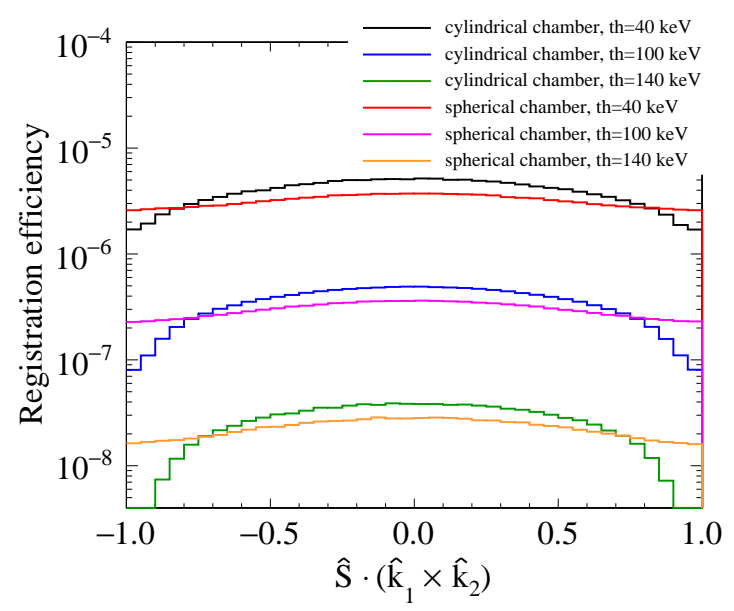

(a)

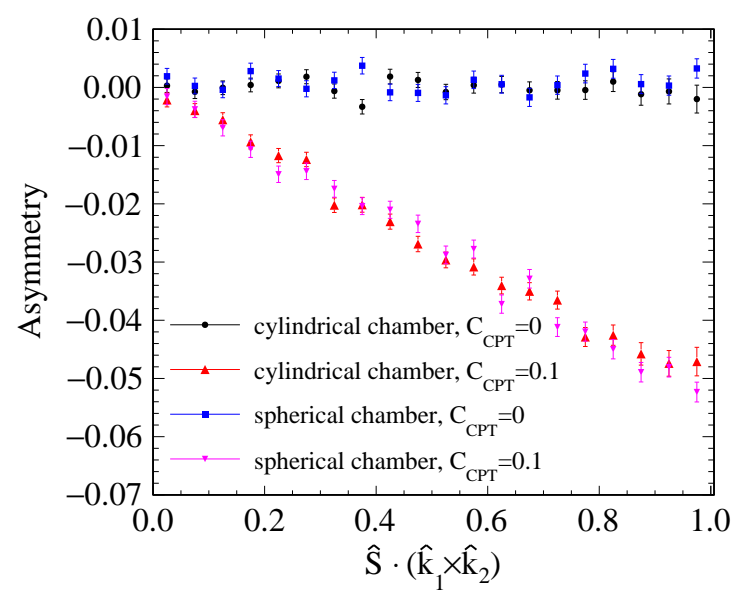

(b)

Figure 3. (a): Total efficiency of registration ortho-positronium (o-Ps) $\rightarrow 3 \gamma$ events in J-PET as a function of the $\vec{S} \cdot\left(\overrightarrow{k_{1}} \times \overrightarrow{k_{2}}\right)$ angular correlation obtained in a MC simulation. The curves present efficiencies in the case of two different geometries of the positronium production chamber: cylindrical and spherical as well as for three values of energy deposition threshold for single $\gamma$ detection. (b): Asymmetry of the $\vec{S} \cdot\left(\overrightarrow{k_{1}} \times \overrightarrow{k_{2}}\right)$ distribution for the two chamber geometries in cases of no CPT violation and an exaggerated violation at the $10 \%$ level assumed in the simulations.

Figure $3 \mathrm{~b}$ shows examples of asymmetries of the $\mathcal{O}_{C P T}$ operator defined as in Equation (2) using the two positronium production chamber geometries considered in this work, for the cases of no asymmetry assumed in the MC simulations and of CPT violation at a level of $10 \%\left(C_{C P T}=0.1\right)$, exaggerated for better visibility. These results were obtained with a simulation of $10^{13}$ positrons from a ${ }^{22} \mathrm{Na}$ source. It is visible that the $\vec{S} \cdot\left(\overrightarrow{k_{1}} \times \overrightarrow{k_{2}}\right)$ angular correlation is not sensitive to the geometry of the positronium annihilation region. Not only are the asymmetries detected using the cylindrical and spherical chambers in good agreement, but also the $A\left(\mathcal{O}_{C P T}\right)$ distribution obtained in absence of simulated CPT violation does not reveal signs of a false asymmetry in any of the cases.

These simulations confirm the robustness of the $\vec{S} \cdot\left(\overrightarrow{k_{1}} \times \overrightarrow{k_{2}}\right)$ angular correlation as an observable of discrete symmetry tests. While potentially sensitive to genuine effects of CPT violation, its definition allows to cancel out many geometrical effects related to the measurement setup. For this reason, this correlation has been favored over the ostensibly simpler operator $\vec{S} \cdot \overrightarrow{k_{1}}$ in the past measurements, even though all of the previous experiments focusing on $\vec{S} \cdot\left(\overrightarrow{k_{1}} \times \overrightarrow{k_{2}}\right)$ were in principle capable of studying the $\vec{S} \cdot \overrightarrow{k_{1}}$ correlation as well. Later on, we discuss the experimental differences between these two correlations.

\subsection{The Correlation between o-PS Spin and Most Energetic Photon}

As discussed in the previous Section, out of the two angular correlations sensitive to discrete symmetries' violation in absence of ortho-positronium tensor polarization (operators 1 and 2 in Table 1), the operator $\vec{S} \cdot\left(\overrightarrow{k_{1}} \times \overrightarrow{k_{2}}\right)$ has already been studied in several experiments. On the contrary, the 1st 
operator which is a simple projection of the most energetic photon momentum onto the direction of spin of the decaying ortho-positronium atom has never been measured to date despite its sensitivity to both CP and CPT-violating effects.

The reason lies in its simpler construction which makes its distribution prone to spurious effects and thus experimentally more challenging. While its usage as an observable of a CP and CPT test requires strict control of the impact of the measurement setup geometry on the observed asymmetry, here we argue that smooth efficiency curves offered by the J-PET detector in conjunction with detailed MC simulations may allow for the first measurement of the $\vec{S} \cdot \overrightarrow{k_{1}}$ operator.

Similarly as in Equation (1), it is convenient to introduce normalization of the photon momentum into the definition of the angular correlation operator:

$$
\mathcal{O}_{C P T}^{\prime}=\vec{S} \cdot \overrightarrow{k_{1}} /\left|\overrightarrow{k_{1}}\right|
$$

Figure 4a presents the efficiency of J-PET to o-Ps $\rightarrow 3 \gamma$ events with a given value of $\mathcal{O}_{C P T}^{\prime}$ evaluated with the toy MC simulation in a similar manner as described in Section 3.2. A comparison with Figure 3a immediately reveals the challenge posed by usage of this operator. In this case, the efficiency curves contain a modulation which is not symmetric as a function of $\mathcal{O}_{C P T}^{\prime}$. Moreover, this effect is magnified with increasing value of the energy deposition threshold for $\gamma$ detection. This energy dependence originates from the choice of the most energetic photon which introduces a correlation with the kinematical configuration of a given o-Ps $\rightarrow 3 \gamma$ decay. This phenomenon is absent in case of $\vec{S} \cdot\left(\overrightarrow{k_{1}} \times \overrightarrow{k_{2}}\right)$ because the geometrical entity used therein (orientation of the decay plane) is agnostic of the kinematics of a particular annihilation event and thus also of the energy-based choice of photons.

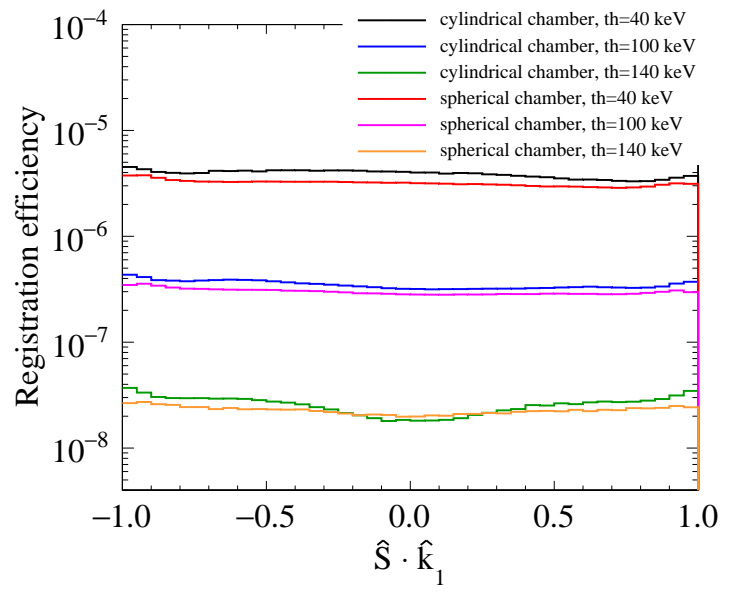

(a)

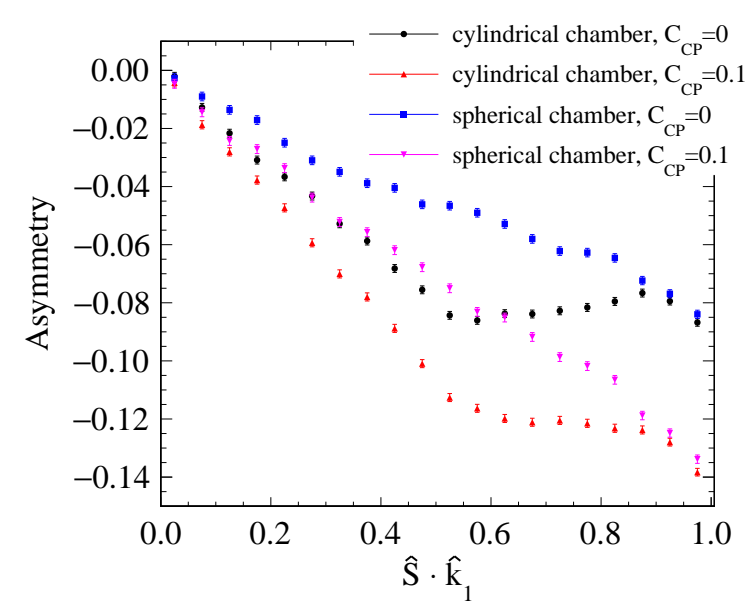

(b)

Figure 4. (a): Total efficiency of registration o-Ps $\rightarrow 3 \gamma$ events in J-PET as a function of the $\vec{S} \cdot \overrightarrow{k_{1}}$ angular correlation obtained in a MC simulation. The curves present efficiencies in case of two different geometries of the positronium production chamber: cylindrical and spherical as well as for three values of energy deposition threshold for single $\gamma$ detection. (b): Asymmetry of the $\vec{S} \cdot \overrightarrow{k_{1}}$ distribution for the two chamber geometries in cases of no CPT violation and an exaggerated violation at the $10 \%$ level assumed in the simulations.

Successful use of the $\mathcal{O}_{C P T}^{\prime}$ operator as a probe of $\mathrm{CP}$ and $\mathrm{CPT}$ violation therefore requires two factors: (i) maintaining the energy deposition threshold as low as possible, (ii) reducing the spurious asymmetries originating from asymmetric and energy-dependent efficiency to a low and well-understood level.

The latter can be achieved by manipulation of the geometry of positronium production medium. As displayed in the right panel of Figure 4, although with both simulated setups the $\vec{S} \cdot \hat{k_{1}}$ a significant asymmetry appears even in case of no CPT violation assumed in the simulations (where possible 
violation is introduced in a similar manner as described in Section 3.2), usage of the spherical vacuum chamber results in a simpler dependence of the false asymmetry on the value of $\mathcal{O}_{C P T}^{\prime}$ which is easier to parameterize. Additionally, two independent measurements with different chambers would allow for discrimination between the setup-specific false asymmetry and a possible genuine effect as well as for extraction of the latter. It is important to stress that while the results presented in this work are based on a toy MC simulation, the actual experiments will be augmented with simulations of the full setup based on the Geant 4 package, which are currently being commissioned.

\section{Perspectives for J-PET Sensitivity to the Cpt Violation Effects}

The J-PET setup featuring the cylindrical annihilation chamber is already in operation. If a conservative photon detection threshold of $100 \mathrm{keV}$ is assumed, it can be estimated from the efficiency curve presented in the left panel of Figure 3 that with a $10 \mathrm{MBq}$ positron source J-PET can record about $8.5 \times 10^{4} \mathrm{o}-\mathrm{Ps} \rightarrow 3 \gamma$ annihilations per day of measurement.

The major expected background source is constituted by direct $e^{+} e^{-} \rightarrow 2 \gamma$ annihilations where a Compton scattered primary photon interacts again in another detection module of J-PET, creating a spurious photon detection interpreted as the third primary annihilation photon. Such events, however, are easily discerned by considering a time-of-flight hypothesis of a photon traveling directly between each pair among three recorded $\gamma$ interactions. Other background sources arise mostly from pick-off annihilations of positrons constituting the positronium atom. These however, either result in a $2 \gamma$ annihilation leading to the case described above or require a direct $e^{+} e^{-} \rightarrow 3 \gamma$ annihilation, suppressed with respect to o-Ps $\rightarrow 3 \gamma$ by a factor of about $10^{-3}$. A comprehensive discussion of physical background sources can be found in References [3,50].

It is noteworthy that even if the dominant background component (direct $2 \gamma$ annihilation with a secondary photon scattering) cannot be fully reduced in the final event sample, the symmetrical construction and even efficiency of the detector assure that this kind of artificial three-photon event should not produce an asymmetry in the distributions of the operators defined in Equations (1) and (3).

The achievable sensitivity for the CPT violation parameter $C_{C P T}$ must include the analyzing power of the employed setup which is dominated by statistical polarization corresponding to the estimated $\mathrm{o}-\mathrm{Ps}$ spin and with a ${ }^{22} \mathrm{Na}$ source amounts to about 0.4. Assuming that the MC-simulated events required for the $C_{C P T}$ extraction procedure described in Section 3.2 can be generated in ample statistics, the sensitivity to the CPT violation parameter is approximately described by the statistical uncertainty on the number $N$ of recorded o-Ps $\rightarrow 3 \gamma$ events and the analyzing power $S$ as $\sigma\left(C_{C P T}\right) \approx N^{-\frac{1}{2}} / S$. J-PET sets as its goal to reach the unprecedented sensitivity at the level beyond $10^{-3}$, which requires $N>6.3 \times 10^{6}$ of event candidates. With the expected rate estimated above, this goal can be achieved with about three months of measurement.

Although J-PET is well suited for extended periods of continuous measurement, further improvements of the efficiency of both positronium production and $3 \gamma$ events detection are necessary in order to allow for reaching the sensitivity of CPT symmetry tests discussed in this work beyond the level of $10^{-4}$.

The two upgrades already being commissioned comprise the spherical vacuum chamber for positronium production and spin estimation and a new layer of detection modules with a fully digital readout. The spherical chamber, in addition to the advantages discussed in Sections 3.2 and 3.3, is expected to increase the fraction of positrons from the $\beta^{+}$source mounted at its center by a factor of about 1.5 with respect to the cylindrical geometry.

This is because the most sensitive region of the J-PET detector spans in the central region along its $\mathrm{Z}$ axis, corresponding to $|z|<8 \mathrm{~cm}$ [36]. Outside of this volume, registration probability for $3 \gamma$ events drops rapidly, therefore in case of the cylindrical chamber only positrons emitted from the $\beta^{+}$ source into a solid angle of about $2.2 \pi$ have a chance to form positronium whose annihilation can be recorded. Therefore, only $55 \%$ of emitted positrons may produce recordable positronium. With the 
spherical geometry, over $80 \%$ of isotropically-emitted positrons will reach the porous medium in the most sensitive region of the detector.

The second upgrade is constituted by insertion of a new system of 312 plastic scintillator strips arranged in 24 densely-packed modules as the innermost layer of the detector as visualized in the right panel of Figure 1. The new fully digital readout system based on silicon photomultipliers is expected to improve time resolution of $\gamma$ interaction recording, crucial for the trilaterative reconstruction of o-Ps annihilations and thus for the event-by-event spin estimation resolution [46]. Moreover, presence of the additional detection layer will increase single photon registration probability by a factor of about 3 , leading to a $27 \times$ enhancement of the total o-Ps $\rightarrow 3 \gamma$ recording efficiency.

The two aforementioned upgrades account for a total improvement of the rate of recording of o-Ps $\rightarrow 3 \gamma$ events by a factor of about 40 with respect to the present setup without using larger $\beta^{+}$source activities. Moreover, the upgrades are not expected to change the signal to background proportion as the enhanced positronium formation and photon detection would both scale the signal and background rates uniformly. Therefore, with the future upgraded setup, J-PET can realistically increase the collected statistics by a factor of 100 required to reach the sensitivity level of $10^{-5}$ with only a moderate increase or source activity or duration of measurement.

It is worth mentioning that the set of discrete symmetry tests possible with J-PET presented in this work is not exhaustive. A second class of angular correlation operators may be defined using the momenta of annihilation photons and their electromagnetic polarization rather than positronium spin direction [3]. Notably, neither of such correlations have been measured to date due to the incapability of the previous positronium experiments to measure photon polarization. Due to the detection principle based on Compton interaction, J-PET is the first detector able to provide a measurement of such angular correlations involving photon electromagnetic polarization $[3,28]$ and experiments towards this end are already ongoing [51,52].

\section{Conclusions}

The J-PET detector is capable of performing tests of the CP and CPT symmetry by determination of the distributions of the angular correlations between ortho-positronium spin and annihilation photons in the o-Ps $\rightarrow 3 \gamma$ process. Preliminary MC simulations demonstrate that the current experimental setup may reach the sensitivity of $10^{-4}$ for the CPT violation parameter in the measurement using the $\vec{S} \cdot\left(\overrightarrow{k_{1}} \times \overrightarrow{k_{2}}\right)$ operator as well as set the first measurement of the $\vec{S} \cdot \overrightarrow{k_{1}}$ operator thanks to smooth response of the detector in function of the angular correlations and good control over spurious asymmetries. Future upgrades of the detector and the positronium formation chamber are expected to provide an about 40-fold increase of statistics in the same measurement time, allowing the discrete symmetry tests with J-PET to reach the sensitivity of $10^{-5}$.

Funding: This research was funded by The Polish National Center for Research and Development through grant INNOTECH-K1/IN1/64/159174/NCBR/12, the Foundation for Polish Science through the MPD and TEAM POIR.04.04.00-00-4204/17 programmes, the National Science Centre of Poland through grants No. 2016/21/B/ST2/01222 and 2017/25/N/NZ1/ 00861, the Ministry for Science and Higher Education through grants No. 6673/IA/SP/2016, 7150/E-338/SPUB/2017/1, 7150/E-338/M/2017 and 7150/E-338/M/2018.

Conflicts of Interest: The author declares no conflict of interest.

\section{References}

1. Wu, C.S.; Ambler, E.; Hayward, R.W.; Hoppes, D.D.; Hudson, R.P. Experimental Test of Parity Conservation in Beta Decay. Phys. Rev. 1957, 105, 1413-1415. [CrossRef]

2. Christenson, J.H.; Cronin, J.W.; Fitch, V.L.; Turlay, R. Evidence for the $2 \pi$ Decay of the $K_{2}^{0}$ Meson. Phys. Rev. Lett. 1964, 13, 138-140. [CrossRef]

3. Moskal, P.; Alfs, D.; Bednarski, T.; Białas, P.; Czerwiński, E.; Curceanu, C.; Gajos, A.; Głowacz, B.; Gorgol, M.; Hiesmayr, B.; et al. Potential of the J-PET detector for studies of discrete symmetries in decays of positronium atom-A purely leptonic system. Acta Phys. Polon. 2016, 47, 509. [CrossRef] 
4. Mills, A.P.; Berko, S. Search for C Nonconservation in Electron-Positron Annihilation. Phys. Rev. Lett. 1967, 18, 420-425. [CrossRef]

5. Bernreuther, W.; Low, U.; Ma, J.P.; Nachtmann, O. How to Test CP, T and CPT Invariance in the Three Photon Decay of Polarized $s$ Wave Triplet Positronium. Z. Phys. 1988, 41, 143-158. [CrossRef]

6. Yamazaki, T.; Namba, T.; Asai, S.; Kobayashi, T. Search for CP Violation in Positronium Decay. Phys. Rev. Lett. 2010, 104, 083401. [CrossRef] [PubMed]

7. Vetter, P.A.; Freedman, S.J. Search for CPT-Odd Decays of Positronium. Phys. Rev. Lett. 2003, 91, 263401. [CrossRef] [PubMed]

8. Vetter, P.A.; Freedman, S.J. Branching-ratio measurements of multiphoton decays of positronium. Phys. Rev. A 2002, 66, 052505. [CrossRef]

9. Matsumoto, T.; Chiba, M.; Hamatsu, R.; Hirose, T.; Yang, J.; Yu, J. Measurement of five-photon decay in orthopositronium. Phys. Rev. A 1996, 54, 1947-1951. [CrossRef] [PubMed]

10. von Busch, H.; Thirolf, P.; Ender, C.; Habs, D.; Köck, F.; Schulze, T.; Schwalm, D. Measurement of the decay $e^{+} e^{-} \rightarrow 4 \gamma$ at rest. Phys. Lett. B 1994, 325, 300-307. [CrossRef]

11. Branco, G.C.; González Felipe, R.; Joaquim, F.R. Leptonic CP violation. Rev. Mod. Phys. 2012, 84, 515-565. [CrossRef]

12. Inami, K.; Abe, K.; Abe, K.; Abe, R.; Abe, T.; Adachi, I.; Aihara, H.; Akatsu, M.; Asano, Y.; Aso, T.; et al. Search for the electric dipole moment of the $\tau$ lepton. Phys. Lett. B 2003, 551, 16-26. [CrossRef]

13. Abe, K.; Akutsu, R.; Ali, A.; Amey, J.; Andreopoulos, C.; Anthony, L.; Antonova, M.; Aoki, S.; Ariga, A.; Ashida, Y.; et al. Search for $C P$ Violation in Neutrino and Antineutrino Oscillations by the T2K Experiment with $2.2 \times 10^{21}$ Protons on Target. Phys. Rev. Lett. 2018, 121, 171802. [CrossRef]

14. Acero, M.A.; Adamson, P.; Alion, L.A.T.; Allakhverdian, V.; Antoshkin, N.A.A.; Arrieta-Diaz, E.; Back, A.A.A.; Backhouse, C.; Balashov, M.B.N.; Bambah, B.A.; et al. New constraints on oscillation parameters from $v_{e}$ appearance and $v_{\mu}$ disappearance in the NOvA experiment. Phys. Rev. D 2018, 98, 032012. [CrossRef]

15. Abe, K.; Akutsu, R.; Ali, A.; Alt, C.; Andreopoulos, C.; Anthony, L.; Antonova, M.; Aoki, S.; Ariga, A.; Asada, Y.; et al. Constraint on the matter-antimatter symmetry-violating phase in neutrino oscillations. Nature 2020, 580, 339-344, doi:10.1038/s41586-020-2177-0. [CrossRef]

16. Kostelecký, V.A.; Vargas, A.J. Lorentz and CPT tests with hydrogen, antihydrogen, and related systems. Phys. Rev. 2015, 92, 056002, doi:10.1103/PhysRevD.92.056002. [CrossRef]

17. Vargas, A.J. Overview of the Phenomenology of Lorentz and CPT Violation in Atomic Systems. Symmetry 2019, 11, 1433. [CrossRef]

18. Moskal, P.; Moskal, P.; Niedźwiecki, S.; Bednarski, T.; Czerwiński, E.; Kubicz, E.; Moskal, I.; Pawlik-Niedźwieckaa, M.; Sharmaa, N.G.; Silarski, M.; et al. Test of a single module of the J-PET scanner based on plastic scintillators. Nucl. Instrum. Meth. 2014, 764, 317-321, doi:10.1016/j.nima.2014.07.052. [CrossRef]

19. Moskal, P.; Zoń, N.; Bednarski, T.; Białas, P.; Czerwiński, E.; Gajos, A.; Kamińska, D.; Kapłon, Ł.; Kochanowski, A.; Korcyl, G.; et al. A novel method for the line-of-response and time-of-flight reconstruction in TOF-PET detectors based on a library of synchronized model signals. Nucl. Instrum. Meth. A 2015, 775, 54-62, doi:10.1016/j.nima.2014.12.005. [CrossRef]

20. Niedźwiecki, S.; Białas, P.; Curceanu, C.; Czerwiński, E.; Dulski, K.; Gajos, A.; Głowacz, B.; Gorgol, M.; Hiesmayr, B.C.; Jasińska, B.; et al. J-PET: A new technology for the whole-body PET imaging. Acta Phys. Polon. 2017, 48, 1567, doi:10.5506/APhysPolB.48.1567. [CrossRef]

21. Kowalski, P.; Wiślicki, W.; Shopa, R.Y.; Raczyński, L.; Klimaszewski, K.; Curcenau, C.; Czerwiński, E.; Dulski, K.; Gajos, A.; Gorgol, M. Estimating the NEMA characteristics of the J-PET tomograph using the GATE package. Phys. Med. Biol. 2018, 63, 165008, doi:10.1088/1361-6560/aad29b. [CrossRef] [PubMed]

22. Moskal, P. Towards total-body modular PET for positronium and quantum entanglement imaging. In Proceedings of the 2018 IEEE Nuclear Science Symposium and Medical Imaging Conference Proceedings (NSS/MIC), Sydney, Australia, 10-17 November 2018; pp. 1-4.

23. Moskal, P.; Stępień, E.Ł. Prospects and clinical perspectives of total-body PET imaging using plastic scintillators. PET Clin. 2020, in press.

24. Moskal, P.; Kisielewska, D.; Curceanu, C.; Czerwiński, E.; Dulski, K.; Gajos, A.; Gorgol, M.; Hiesmayr, B.; Jasińska, B.; Kacprzak, K. Feasibility study of the positronium imaging with the J-PET tomograph. Phys. Med. Biol. 2019, 64, 055017, doi:10.1088/1361-6560/aafe20. [CrossRef] [PubMed] 
25. Moskal, P.; Jasińska, B.; Stępień, E.Ł.; Bass, S.D. Positronium in medicine and biology. Nat. Rev. Phys. 2019, 1, 527-529. [CrossRef]

26. Moskal, P.; Kisielewska, D.; Bura, Z.; Chhokar, C.; Curceanu, C.; Czerwiński, E.; Dadgar, M.; Dulski, K.; Gajewski, J.; Gajos, A.; et al. Performance assessment of the $2 \gamma$ positronium imaging with the total-body PET scanners. EJNMMI Phys. 2020, 7, 44. [CrossRef] [PubMed]

27. Hiesmayr, B.; Moskal, P. Genuine Multipartite Entanglement in the 3-Photon Decay of Positronium. Sci. Rep. 2017, 7, 15349, doi:10.1038/s41598-017-15356-y. [CrossRef]

28. Moskal, P.; Krawczyk, N.; Hiesmayr, B.C.; Bała, M.; Curceanu, C.; Czerwiński, E.; Dulski, K.; Gajos, A.; Gorgol, M.; Grande, R.D.; et al. Feasibility studies of the polarization of photons beyond the optical wavelength regime with the J-PET detector. Eur. Phys. J. C 2018, 78, 970, doi:10.1140/epjc/s10052-018-6461-1. [CrossRef]

29. Hiesmayr, B.C.; Moskal, P. Witnessing Entanglement In Compton Scattering Processes Via Mutually Unbiased Bases. Sci. Rep. 2019, 9, 8166, doi:10.1038/s41598-019-44570-z. [CrossRef]

30. Pałka, M.; Strzempek, P.; Korcyl, G.; Bednarski, T.; Niedźwiecki, S.; Białas, P.; Czerwiński, E.; Dulski, K.; Gajos, A.; Głowacz, B.; et al. Multichannel FPGA based MVT system for high precision time (20 ps RMS) and charge measurement. JINST 2017, 12, P08001, doi:10.1088/1748-0221/12/08/P08001. [CrossRef]

31. Pałka, M.; Bednarski, T.; Białas, P.; Czerwiński, E.; Kapłon, L.; Kochanowski, A.; Korcyl, G.; Kowal, J.; Kowalski, P.; Kozik, T.; et al. A novel method based solely on FPGA units enabling measurement of time and charge of analog signals in Positron Emission Tomography. Bio-Algorithms Med-Syst. 2014, 10, 41-45, doi:10.1515/bams-2013-0104. [CrossRef]

32. Korcyl, G.; Alfs, D.; Bednarski, T.; Białas, P.; Czerwiński, E.; Dulski, K.; Gajos, A.; Głowacz, B.; Jasińska, B.; Kamińska, D.; et al. Sampling FEE and Trigger-less DAQ for the J-PET Scanner. Acta Phys. Polon. 2016, 47, 491, doi:10.5506/APhysPolB.47.491. [CrossRef]

33. Korcyl, G.; Białas, P.; Curceanu, C.; Czerwiński, E.; Dulski, K.; Flak, B.; Gajos, A.; Głowacz, B.; Gorgol, M.; Hiesmayr, B.C.; et al. Evaluation of Single-Chip, Real-Time Tomographic Data Processing on FPGA SoC Devices. IEEE Trans. Med. Imaging 2018, 37, 2526-2535. [CrossRef] [PubMed]

34. Sharma, S. Time Over Threshold as a measure of energy response of plastic scintillators used in the J-PET detector. EPJ Web Conf. 2019, 199, 05014. [CrossRef]

35. Sharma, S.; Chhokar, J.; Curceanu, C.; Czerwinski, E.; Dadgar, M.; Dulski, K.; Gajewski, J.; Gajos, A.; Gorgol, M.; Gupta-Sharma, N.; et al. Estimating relationship between the Time Over Threshold and energy loss by photons in plastic scintillators used in the J-PET scanner. EJNMMI-Phys. 2020, 7, 39. [CrossRef] [PubMed]

36. Gajos, A.; Curceanu, C.; Czerwiński, E.; Dulski, K.; Gorgol, M.; Gupta-Sharma, N.; Hiesmayr, B.C.; Jasińska, B.; Kacprzak, K.; Kapłon, Ł.; et al. Feasibility Study of the Time Reversal Symmetry Tests in Decay of Metastable Positronium Atoms with the J-PET Detector. Adv. High Energy Phys. 2018, 2018, 8271280, doi:10.1155/2018/8271280. [CrossRef]

37. Korcyl, G.; Moskal, P.; Bednarski, T.; Białas, P.; Czerwiński, E.; Kapłon, L.; Kochanowski, A.; Kowal, J.; Kowalski, P.; Kozik, T.; et al. Trigger-less and reconfigurable data acquisition system for positron emission tomography. Bio-Algorithms Med-Syst. 2014, 10, 37-40. [CrossRef]

38. Krzemień, W.; Gajos, A.; Gruntowski, A.; Stola, K.; Trybek, D.; Bednarski, T.; Białas, P.; Czerwiński, E.; Kamińska, D.; Kapłon, L.; et al. Analysis framework for the J-PET scanner. Acta Phys. Polon. A 2015, 127, 1491-1494, doi:10.12693/APhysPolA.127.1491. [CrossRef]

39. Krzemień, W.; Alfs, D.; Bialas, P.; Czerwinski, E.; Gajos, A.; Glowacz, B.; Jasinska, B.; Kaminska, D.; Korcyl, G.; Kowalski, P.; et al. Overview of the software architecture and data flow for the J-PET tomography device. Acta Phys. Polon. B 2016, 47, 561, doi:10.5506/APhysPolB.47.561. [CrossRef]

40. Krzemien, W.; Gajos, A.; Kacprzak, K.; Rakoczy, K.; Korcyl, G. J-PET Framework: Software platform for PET tomography data reconstruction and analysis. SoftwareX 2020, 11, 100487, doi:10.1016/j.softx.2020.100487. [CrossRef]

41. Moskal, P.; Rundel, O.; Alfs, D.; Bednarski, T.; Białas, P.; Czerwiński, E.; Gajos, A.; Giergiel, K.; Gorgol, M.; Jasińska, B.; et al. Time resolution of the plastic scintillator strips with matrix photomultiplier readout for J-PET tomograph. Phys. Med. Biol. 2016, 61, 2025, doi:10.1088/0031-9155/61/5/2025. [CrossRef]

42. Skalsey, M.; Van House, J. First test of CP invariance in the decay of positronium. Phys. Rev. Lett. 1991, 67, 1993-1996. [CrossRef] [PubMed] 
43. Arbic, B.K.; Hatamian, S.; Skalsey, M.; Van House, J.; Zheng, W. Angular Correlation Test of CPT in Polarized Positronium. Phys. Rev. 1988, 37, 3189-3194. [CrossRef] [PubMed]

44. Bernreuther, W.; Low, U.; Nachtmann, O. Possible tests of CP invariance with polarized positronium. Hyperfine Interact. 1989, 44, 139-145. [CrossRef]

45. Mohammed, M.; Białas, P.; Curceanu, C.; Czerwiński, E.; Dulski, K.; Gajos, A.; Głowacz, B.; Gorgol, M.; Hiesmayr, B.C.; Jasinska, B.; et al. A method to produce linearly polarized positrons and positronium atoms with the J-PET detector. Acta Phys. Polon. 2017, 132, 1486-1489. [CrossRef]

46. Gajos, A.; Kamińska, D.; Czerwiński, E.; Alfs, D.; Bednarski, T.; Białas, P.; Głowacz, B.; Gorgol, M.; Jasińska, B.; Kapłon, L.; et al. Trilateration-based reconstruction of ortho-positronium decays into three photons with the J-PET detector. Nucl. Instrum. Meth. 2016, 819, 54-59, doi:10.1016/j.nima.2016.02.069. [CrossRef]

47. Gajos, A. Studies of Ortho-Positronium Decays into Three Photons with the J-PET Detector. Acta Phys. Polon. 2020, 137, 126-129. [CrossRef]

48. Abe, K.; Abt, I.; Ahn, C.J.; Akagi, T.; Allen, N.J.; Ash, W.W.; Aston, D.; Baird, K.G.; Baltay, C.; Band, H.R.; et al. First Measurement of the T-Odd Correlation between the $Z^{0}$ Spin and the Three-Jet Plane Orientation in Polarized $Z^{0}$ Decays into Three Jets. Phys. Rev. Lett. 1995, 75, 4173-4177. [CrossRef]

49. Berestetskii, V.B.; Lifshitz, E.M.; Pitaevskii, L.P. Relativistic Quantum Theory, 1st ed.; Pergamon Press: Oxford, UK; New York, NY, USA, 1971.

50. Kamińska, D.; Gajos, A.; Czerwiński, E.; Alfs, D.; Bednarski, T.; Białas, P.; Curceanu, C.; Dulski, K.; Głowacz, B.; Gupta-Sharma, N.; et al. A feasibility study of ortho-positronium decays measurement with the J-PET scanner based on plastic scintillators. Eur. Phys. J. 2016, 76, 1-14, doi:10.1140/epjc/s10052-016-4294-3. [CrossRef]

51. Raj, J.; Gajos, A.; Curceanu, C.; Czerwiński, E.; Dulski, K.; Gorgol, M.; Gupta-Sharma, N.; Hiesmayr, B.C.; Jasińska, B.; Kacprzak, K.; et al. A feasibility study of the time reversal violation test based on polarization of annihilation photons from the decay of ortho-Positronium with the J-PET detector. Hyperfine Interact. 2018, 239, 56, doi:10.1007/s10751-018-1527-x. [CrossRef]

52. Raj, J.; Kisielewska, D.; Czerwiński, E. J-PET Monte Carlo Simulations for Time-Reversal Symmetry Test in Ortho-Positronium Decay. Acta Phys. Polon. 2020, 137, 137-139. [CrossRef]

(C) 2020 by the authors. Licensee MDPI, Basel, Switzerland. This article is an open access article distributed under the terms and conditions of the Creative Commons Attribution (CC BY) license (http:/ / creativecommons.org/licenses/by/4.0/). 